\title{
Aplicação do software Excel como ferramenta de ensino para resolução de treliças planas utilizando elementos finitos de barras
}

Charles Jaster de Oliveira iD chjaster@up.edu.br Universidade Positivo (UP)

Luna Ollin Steffen iD luna_steffen@hotmail.com Universidade Tecnológica do Paraná (UTFPR)

Guilherme Macedo Vogel iD rsgguilherme@gmail.com Universidade Positivo (UP)

Raphael Brandalise Nunes iD leandro.filho@ifce.edu.br Universidade Positivo (UP)

Gabriel Martins dos Santos iD gabriel_martins_97@ hotmail.comUniversidade Positivo (UP)

\section{Application of the Excel software as a teaching tool for resolution of flat trusses using the finite elements method}

\section{Aplicación del software Excel como herramienta de enseñanza para resolución de enrejados planos utilizando el método de los elementos finitos}

\section{Application du logiciel Excel en tant qu'outil pédagogique pour la résolution de treillis plats à l'aide de la méthode des éléments finis}

\section{Resumo}

A determinação de esforços axiais e deslocamentos são indispensáveis para a análise e a compreensão de uma treliça. Para tais determinações, há uma constante busca de métodos que possam facilitar os cálculos. Através do método dos elementos finitos (MEF), o presente artigo propõe um modelo de planilha eletrônica, desenvolvida no Microsoft Exce/®, capaz de calcular os esforços das barras que constituem a treliça plana, bem como os deslocamentos nodais, quando sujeitas a forças e restrições de deslocamentos. A fim de não somente apresentar números, de modo didático, o modelo também demonstra graficamente os resultados obtidos utilizando o Visual Basic for Applications (VBA), que é

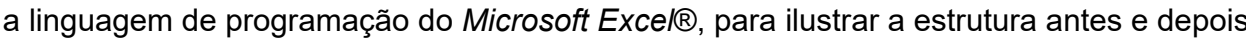
da aplicação das forças, mostrando os esforços de tração e compressão nas barras e o deslocamento dos nós. O uso dessa programação, além de facilitar os cálculos, também se faz importante didaticamente, visto que é uma ferramenta capaz de confirmar os resultados que são obtidos a partir de métodos tradicionais, comum nas graduações de engenharia civil, devido a fácil curva de aprendizado e a alta disponibilidade do software.

Palavras-chave: Ferramenta educacional. Planilhas eletrônicas. Treliças planas.

\begin{abstract}
The determination of axial forces and displacements is indispensable for the analysis and understanding of a lattice. For such determinations, there is a constant search for methods that can facilitate and expedite the calculations. Through the Finite Element Method (MEF), the present article proposes a spreadsheet model, developed in Microsoft Excel ${ }^{\circledR}$, capable of calculating the stresses of the bars that constitute the flat truss, as well as the nodal displacements, when subjected to forces and restrictions. In order not only to present numbers, in a didactic way, the model also graphically demonstrates the results obtained using Visual Basic for Applications (VBA), which is the programming language of Microsoft Excel $\circledast$, to illustrate the structure before and after the application of the forces showing tensile and compressive forces on the bars and the displacement of the nodes. The use of programming

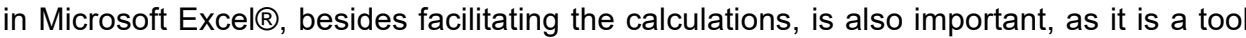
capable of confirming the results obtained through the traditional methods, common in civil engineering graduations and due to its easy learning and the high availability of the software.
\end{abstract}

Keywords: Educational tool. Spreadsheets. Flat trusses. 


\title{
Resumen
}

La determinación de esfuerzos axiales y desplazamientos son indispensables para el análisis y la comprensión de un enrejado. Para tales determinaciones, hay una constante búsqueda de métodos que puedan facilitar y agilizar los cálculos. Por medio del Método de los Elementos Finitos (MEF), el presente trabajo propone un modelo de planilla electrónica, desarrollada en el Microsoft Excel ${ }^{\circledR}$, capaz de calcular los esfuerzos de las barras que constituyen el enrejado plano, así como los desplazamientos nodales, cuando sujetos a fuerzas y restricciones de desplazamientos. Con el fin de no presentar solamente números, de modo didáctico, el modelo también demuestra gráficamente los resultados obtenidos utilizando el Visual Basic for Applications (VBA), que es el lenguaje de programación del Microsoft Excel $\circledast$, para ilustrar la estructura antes y después de la aplicación de las fuerzas enseñando los esfuerzos de tracción y compresión en las barras y el desplazamiento de los nudos. El uso de la programación en el Microsoft Excel® además de facilitar los cálculos, también es importante didácticamente, visto que es una herramienta capaz de confirmar los resultados que son obtenidos vía métodos tradicionales, común en los cursos de grado en ingeniería civil y debido a su fácil curva de aprendizaje y el alta disponibilidad del software.

Palabras-clave: Herramienta educacional. Planillas electrónicas. Enrejados planos.

\begin{abstract}
Résumé
La détermination des efforts axiaux et des déplacements sont indispensables à l'analyse et à la compréhension d'un treillis. Pour ces déterminations, il y a une recherche constante de méthodes qui puissent faciliter et accélérer les calculs. En utilisant la méthode des éléments finis (MEF), cet article propose un modèle de tableur électronique, développé sous Microsoft Excel ${ }^{\circledR}$, capable de calculer les forces des barres qui constituent le treillis plat, ainsi que les déplacements nodaux, lorsqu'ils sont soumis à des forces et à des restrictions de déplacement. On ne veut pas seulement d'afficher les nombres de manière didactique, mais aussi montrer, de manière graphique, les résultats obtenus. À l'aide de Visual Basic pour Applications (VBA), langage de programmation de Microsoft Excel®, on présente la structure avant et après l'application de forces montrant les forces de traction et de compression exercées sur les barres et le déplacement des nœuds. L'utilisation de la programmation dans Microsoft Excel $\circledast$, en plus de faciliter les calculs, est également importante du point de vue didactique, car c'est un outil capable de confirmer les résultats obtenus par les méthodes traditionnelles, courantes dans les cours de génie civil. En autre, il y a une courbe d'apprentissage facile et haute disponibilité de ce logiciel.
\end{abstract}

Mots-clés : Outil d'apprentissage. Tableur électronique. Treillis plats.

\section{Introdução}

O método dos elementos finitos (MEF) é um método numérico eficiente para a resolução de problemas de engenharia em meios contínuos. Apesar de ter sido idealizado na década de 30, foi somente com o advento da computação que sua prática se tornou viável, pois, sem o uso da tecnologia, demandava uma carga de trabalho extenuante para sua utilização e seus resultados não apresentavam uma precisão que justificasse a substituição do método tradicional de cálculo pelo MEF (GODOI, 2017).

Atualmente, o uso do MEF em softwares tornou-se muito comum, por ter a capacidade de apresentar resultados muito próximos da realidade, além de diminuir custos de projeto, carga de trabalho e facilitar a compreensão e o entendimento de problemas da engenharia (NOGUEIRA; BEZERRA, 2017).

O Excel possui alta disponibilidade no mercado, além de possuir uma interface de fácil compreensão e inúmeros recursos matemáticos (NOGUEIRA; BEZERRA, 2017), fato que auxilia o cálculo dos esforços axiais e os deslocamentos nodais através da programação proposta por este trabalho. 
O objetivo do presente trabalho acadêmico é fazer uso do MEF, através do Microsoft Exce/, para o cálculo dos esforços axiais de cada barra constituinte de uma treliça plana, bem como dos deslocamentos nodais, através de uma ferramenta capaz de tornar o processo de cálculo mais didático, facilitando a aprendizagem e apresentando todo o memorial de cálculo utilizado, além de demonstrar os resultados, também graficamente, por meio da ferramenta Visual Basic for Applications (VBA).

\section{Fundamentação teórica}

No desenvolvimento do presente trabalho utilizou-se, como base principal, o método dos elementos finitos, o qual consiste na ideia de discretização, isto é, em transformar um problema complexo em pequenos problemas simples e, assim, analisar uma estrutura global, em meios contínuos, através das pequenas parcelas que a integram. Tais pequenas parcelas apresentam comportamento de forma semelhante ao meio contínuo original (SORIANO, 2003). A análise por elementos finitos pode ser utilizada para o entendimento de diferentes estruturas, como vigas, placas e cascas (SORIANO, 2003), porém, para este trabalho, o método foi usado para a análise de estruturas treliçadas, sendo realizado um estudo das barras que compõem a treliça, a fim de analisar a estrutura como um todo. As barras são elementos que apresentam seção transversal pequena comparada ao seu comprimento, sujeitas, basicamente, a esforços axiais, tração e compressão. Além disso, elas apresentam algumas premissas que possibilitam os cálculos numéricos, tais como: a soma das forças internas que atuam nas barras é nula; apresenta comportamento elástico, seguindo a lei de Hooke; e as barras não apresentam fendas e sobreposições após serem deformadas (SILVA NETO et al., 2007).

O MEF, no presente trabalho, foi aplicado através do método direto, cuja base é na interpretação física da estrutura em análise. Trata-se de um método limitado a problemas de pequena complexidade. O método consiste na análise da estrutura completa como uma combinação das análises dos respectivos elementos individuais. As matrizes de rigidez desses elementos, correspondendo aos graus de liberdade global da estrutura, calculadas e adicionadas, culminam na matriz de rigidez global (BATHE, 2014). Posteriormente, a solução das equações de equilíbrio, através da combinação dessa matriz global com os vetores de força aplicados, obtém os deslocamentos nodais, que, por fim, são utilizadas para cálculo dos esforços axiais nas barras (MARTHA, 2010).

\section{Metodologia}

Inicialmente, o programa faz uma leitura dos dados de entrada, conforme a Fig.1. Sendo assim, o usuário deve informar:

- As coordenadas nodais;

- Os nós inicial e final de cada barra;

- A área da seção transversal e o módulo de elasticidade de cada barra;

- O tipo de apoio e sua coordenada;

- As forças nodais atuantes e em que nó estão aplicadas;

- A escala a ser utilizada na representação da estrutura deformada. 
Figura 1 - Entrada de dados.

\begin{tabular}{|c|c|c|c|c|c|c|}
\hline \multicolumn{7}{|c|}{ Coordenadas nodais } \\
\hline Nó & 1 & 2 & 3 & 4 & 5 & 6 \\
\hline X_coord & 0,0 & 1,0 & 2,0 & 2,0 & 1,0 & 0,0 \\
\hline Y_coord & 0,0 & 0,0 & 0,0 & 1,0 & 1,0 & 1,0 \\
\hline
\end{tabular}

\begin{tabular}{|c|c|c|c|c|c|c|c|c|c|}
\hline \multicolumn{10}{|c|}{ Informaçōes dos elementos } \\
\hline$\overline{\text { Barra }}$ & 1 & 2 & 3 & 4 & 5 & $\overline{6}$ & 7 & 8 & 9 \\
\hline Nó inicial & 1 & 2 & 3 & 3 & 2 & 2 & 1 & 5 & 4 \\
\hline Nó final & 2 & 3 & 4 & 5 & 5 & 6 & 6 & 6 & 5 \\
\hline A Sec & 10,000 & 10,000 & 10,000 & 10,000 & 10,000 & 10,000 & 10,000 & 10,000 & 10,000 \\
\hline$E$ & $1,000 \mathrm{E}+06$ & $1,000 \mathrm{E}+06$ & $1,000 \mathrm{E}+06$ & $1,000 \mathrm{E}+06$ & $1,000 E+06$ & $1,000 \mathrm{E}+06$ & $1,000 \mathrm{E}+06$ & $1,000 \mathrm{E}+06$ & $1,000 \mathrm{E}+06$ \\
\hline PLOT & 11,111 & 11,111 & 11,111 & 11,111 & 11,111 & 11,111 & 11,111 & 11,111 & 11,111 \\
\hline Escala & 100 & & & & & & & & \\
\hline \multicolumn{3}{|c|}{ Condiçōes de apoio } & & & & & & & \\
\hline No. & 1 & 6 & & & & & & & \\
\hline $\bar{R} x$ & 1 & 1 & & & & & & & \\
\hline R_y & 1 & 1 & & & & & & & \\
\hline \multicolumn{7}{|c|}{ Carregamento } & & & \\
\hline Nó & 1 & 2 & 3 & 4 & 5 & 6 & & & \\
\hline$\overline{P x}$ & 0,00 & 0,00 & 0,00 & 0,00 & 0,00 & 0,00 & & & \\
\hline$P y$ & 0,00 & 0,00 & 0,00 & -100 & $-50,00$ & 0,00 & & & \\
\hline
\end{tabular}

Para a aplicação do método dos elementos finitos na análise de estruturas treliçadas planas foram lidos os dados de entrada, sendo geradas as matrizes de rigidez de cada elemento.

Para o cálculo das matrizes de rigidez elementar, partimos do princípio de que um elemento de treliça pode sofrer deslocamento relativo apenas ao longo do seu eixo longitudinal. Entende-se que é possível determinar apenas dois deslocamentos, referentes aos deslocamentos de seus extremos, ao imaginarmos uma barra de treliça e assumirmos um deslocamento relativo em apenas um de seus extremos, como representado na Fig. 2:

Figura 2 - Representação do deslocamento nodal.

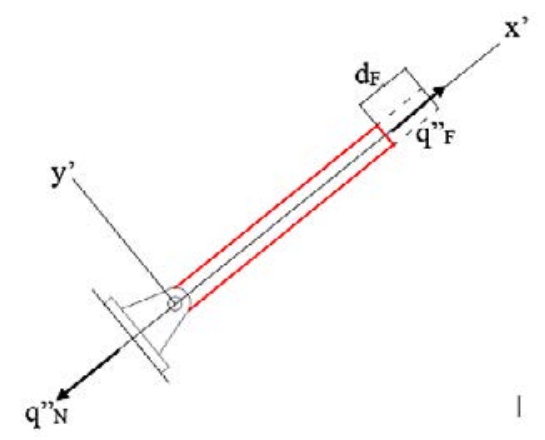

Temos duas forças de mesmo módulo, porém com sinais opostos, devido sua orientação a favor e contra o eixo de coordenadas local. Sendo assim, temos:

$$
\begin{aligned}
& q^{\prime \prime}{ }_{N}=-\frac{A E}{L} d_{F} \\
& q_{F}^{\prime \prime}=\frac{A E}{L} d_{F}
\end{aligned}
$$

Em que A representa a área da seção transversal do elemento, $\mathrm{E}$ o módulo de elasticidade e $\mathrm{L}$ o seu comprimento. Ao invertermos o extremo sujeito ao deslocamento relativo, ação ilustrada pela Fig. 3, concluímos que:

$$
\begin{aligned}
& q_{F}^{\prime}=-\frac{A E}{L} d_{N} \\
& q_{N}^{\prime}=\frac{A E}{L} d_{N}
\end{aligned}
$$


Figura 3 - Representação do deslocamento nodal.

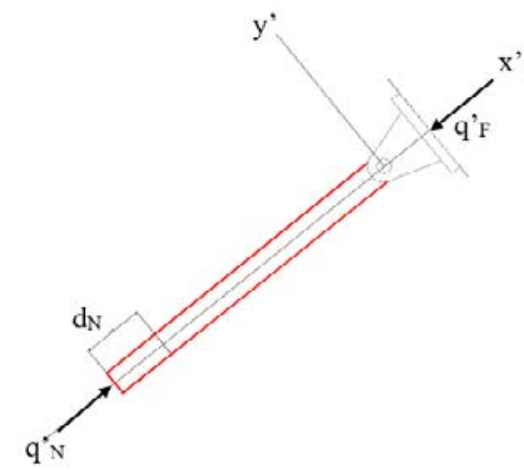

Aplicando o processo de superposição dos efeitos causados pelos deslocamentos, calculados nas equações 1, 2, 3 e 4, nas extremidades, chegamos em:

$$
\begin{aligned}
& q_{N}=q_{N}^{\prime}+q^{\prime \prime}{ }_{N}=\frac{A E}{L} d_{N}-\frac{A E}{L} d_{F} \\
& q_{F}=q_{F}^{\prime}+q_{F}^{\prime \prime}=-\frac{A E}{L} d_{N}+\frac{A E}{L} d_{F}
\end{aligned}
$$

Representando as equações 5 e 6 matricialmente e colocando o termo $\frac{A E}{L}$ em evidência, podemos concluir que a matriz de rigidez elementar em coordenadas locais pode ser representada por:

$$
\left\{\begin{array}{l}
q_{N} \\
q_{F}
\end{array}\right\}=\frac{A E}{L}\left[\begin{array}{cc}
1 & -1 \\
-1 & 1
\end{array}\right]\left\{\begin{array}{l}
d_{N} \\
d_{F}
\end{array}\right\}
$$

Tendo em vista que uma treliça não é composta de uma só barra, mas de várias, é necessário transformar os vetores de deslocamento e de força, definidos segundo o sistema de coordenadas locais, em vetores que seguem o sistema de coordenadas global da estrutura. Para que isso seja possível, devemos determinar Ix e ly, que representam o cosseno dos menores ângulos entre os sentidos positivos do sistema de eixo global ( $x$, y) e o eixo positivo $x$ ' do sistema local, e tais ângulos recebem a denominação de qx e qy, ou seja:

$$
\begin{aligned}
& \lambda_{x}=\cos \theta_{x} \\
& \lambda_{y}=\cos \theta_{y}
\end{aligned}
$$

Figura 4 - Sistema de coordenadas locais.

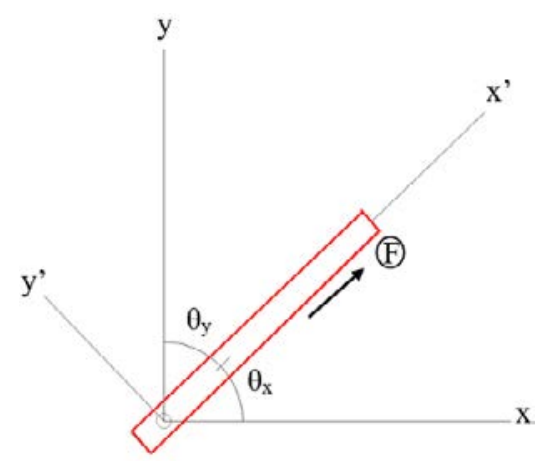

Por meio de relações trigonométricas mostradas na Fig. 4, podemos definir $\lambda_{\mathrm{x}} \mathrm{e}^{\lambda_{\mathrm{y}} \text { como: }}$ 


$$
\begin{aligned}
& \lambda_{x}=\cos \theta_{x}=\frac{X_{F}-X_{N}}{L}=\frac{X_{F}-X_{N}}{\sqrt[2]{\left(X_{F}-X_{N}\right)^{2}+\left(Y_{F}-Y_{N}\right)^{2}}} \\
& \lambda \mathrm{y}=\cos \theta y=\frac{Y_{F}-Y_{N}}{L}=\frac{Y_{F}-Y_{N}}{\sqrt[2]{\left(X_{F}-X_{N}\right)^{2}+\left(Y_{F}-Y_{N}\right)^{2}}}
\end{aligned}
$$

Considerando que as coordenadas globais cada elemento estrutural apresenta 2 graus de liberdade e analisando os deslocamentos separadamente, temos que:

$$
\begin{aligned}
& d_{N}=D_{N x} \lambda_{x}+D_{N y} \lambda_{y} \\
& d_{F}=D_{F x} \lambda_{x}+D_{F y} \lambda_{y}
\end{aligned}
$$

Escrevendo as equações 12 e 13 de forma matricial, obtemos a matriz de transformação do vetor de deslocamentos em coordenadas locais para vetor de deslocamentos em coordenadas globais:

$$
\left\{\begin{array}{l}
d_{N} \\
d_{F}
\end{array}\right\}=\left[\begin{array}{cccc}
\lambda_{x} & \lambda_{y} & 0 & 0 \\
0 & 0 & \lambda_{x} & \lambda_{y}
\end{array}\right]\left\{\begin{array}{l}
D_{N x} \\
D_{N y} \\
D_{F x} \\
D_{F y}
\end{array}\right\}
$$

Com o objetivo de descobrirmos a matriz de transformação do vetor de forças, aplicamos uma força $\mathrm{q}_{\mathrm{N}}$ e $\mathrm{q}_{\mathrm{F}}$ nas duas extremidades de um elemento de barra, assim temos:

$$
\begin{aligned}
& Q_{N x}=q_{N} \cos \theta_{x}=q_{N} \lambda_{x} \\
& Q_{N y}=q_{N} \cos \theta_{y}=q_{N} \lambda_{y} \\
& Q_{F x}=q_{F} \cos \theta_{x}=q_{F} \lambda_{x} \\
& Q_{F y}=q_{F} \cos \theta_{y}=q_{F} \lambda_{y}
\end{aligned}
$$

Escrevendo de forma matricial as equações 15, 16, 17 e 18:

$$
\left\{\begin{array}{l}
Q_{N x} \\
Q_{N y} \\
Q_{F x} \\
Q_{F y}
\end{array}\right\}=\left[\begin{array}{ll}
\lambda_{x} & 0 \\
\lambda_{y} & 0 \\
0 & \lambda_{x} \\
0 & \lambda_{y}
\end{array}\right]\left\{\begin{array}{l}
q_{N} \\
q_{F}
\end{array}\right\}
$$

Realizando as combinações das matrizes obtidas, podemos determinar os esforços e os deslocamentos globais a partir dos esforços e dos deslocamentos locais. Sendo assim, obtemos a matriz de rigidez elementar em coordenadas globais da seguinte forma:

$$
[K]=\left[\begin{array}{ll}
\lambda_{x} & 0 \\
\lambda_{y} & 0 \\
0 & \lambda_{x} \\
0 & \lambda_{y}
\end{array}\right] \frac{A E}{L}\left[\begin{array}{cc}
1 & -1 \\
-1 & 1
\end{array}\right]\left[\begin{array}{cccc}
\lambda_{x} & \lambda_{y} & 0 & 0 \\
0 & 0 & \lambda_{x} & \lambda_{y}
\end{array}\right]
$$

Realizando as multiplicações das matrizes podemos chegar à conclusão de que a matriz de rigidez de uma barra de treliça é definida pela equação 21. Tal matriz é representada na planilha como ilustrado na Fig. 5. 
Aplicação do software Excel como ferramenta de ensino para resolução de treliças planas utilizando elementos finitos de barras

$$
[K]=\frac{A E}{L}\left[\begin{array}{cccc}
\lambda_{x}{ }^{2} & \lambda_{x} \lambda_{y} & -\lambda_{x}{ }^{2} & -\lambda_{x} \lambda_{y} \\
\lambda_{x} \lambda_{y} & \lambda_{y}{ }^{2} & -\lambda_{x} \lambda_{y} & -\lambda_{y}{ }^{2} \\
\lambda_{x}{ }^{2} & -\lambda_{x} \lambda_{y} & \lambda_{x}{ }^{2} & \lambda_{x} \lambda_{y} \\
\lambda_{x} \lambda_{y} & -\lambda_{y}{ }^{2} & \lambda_{x} \lambda_{y} & \lambda_{y}{ }^{2}
\end{array}\right]
$$

Figura 5 - Exemplo de matriz de rigidez elementar calculada pela planilha

\begin{tabular}{|cccc|}
\hline \multicolumn{4}{c}{ Barra 1} \\
TETA & $\mathbf{L}$ & $\mathbf{K}$ & \\
0 & $1,00 \mathrm{E}+00$ & $1,00 \mathrm{E}+07$ & \\
$\mathrm{u} 1$ & $\mathrm{~V} 1$ & $\mathrm{u} 2$ & $\mathrm{v} 2$ \\
$1,00 \mathrm{E}+07$ & $0,00 \mathrm{E}+00$ & $-1,00 \mathrm{E}+07$ & $0,00 \mathrm{E}+00$ \\
$0,00 \mathrm{E}+00$ & $0,00 \mathrm{E}+00$ & $0,00 \mathrm{E}+00$ & $0,00 \mathrm{E}+00$ \\
$-1,00 \mathrm{E}+07$ & $0,00 \mathrm{E}+00$ & $-1,00 \mathrm{E}+07$ & $0,00 \mathrm{E}+00$ \\
$0,00 \mathrm{E}+00$ & $0,00 \mathrm{E}+00$ & $0,00 \mathrm{E}+00$ & $0,00 \mathrm{E}+00$ \\
\hline
\end{tabular}

Após a determinação das matrizes de rigidez de cada barra da treliça, obtidas pela equação 21 , é realizado o processo de acoplamento das matrizes de todos os elementos para a construção da matriz de rigidez global da estrutura, o que é feito através do processo ilustrado na Fig. 6.

Figura 6 - Processo de acoplamento de matrizes

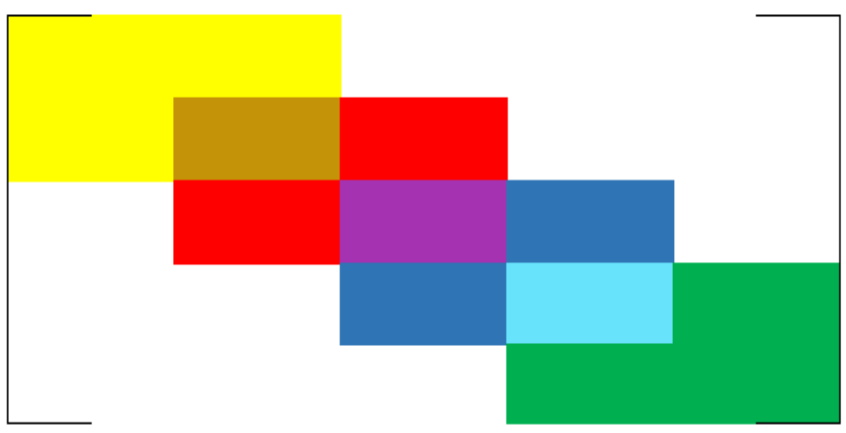

\begin{tabular}{|l|l|}
\hline & Matriz de rigidez barra 1 \\
\hline & Intersecção entre barras 1 e 2 \\
\hline & Matriz de rigidez barra 2 \\
\hline & Intersecção entre barras 2 e 3 \\
\hline & Matriz de rigidez barra 3 \\
\hline & Intersecção entre barras 3 e 4 \\
\hline & Matriz de rigidez barra 4 \\
\hline
\end{tabular}

Determinada a matriz de rigidez global, exemplificada na Fig. 7, seguiu-se o processo de cálculo através da multiplicação da matriz de rigidez pelo vetor deslocamento, o que gerou uma matriz resultante. Tal matriz é submetida ao processo de inversão de matrizes e, posteriormente, é multiplicada pelo vetor das forças nodais aplicadas, e assim podemos determinar os valores dos deslocamentos dos nós.

Figura 7 - Exemplo de matriz de rigidez global gerada pela planilha

\begin{tabular}{|c|c|c|c|c|c|c|c|c|c|c|c|}
\hline \multicolumn{12}{|c|}{ 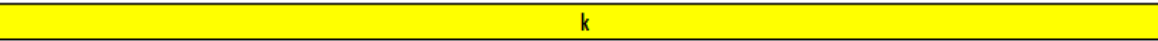 } \\
\hline$u 1$ & v1 & $\mathrm{u} 2$ & v2 & u3 & 43 & 44 & v4 & u5 & v5 & 46 & 46 \\
\hline $1,00 E+06$ & 6,13E-11 & $-1,00 E+06$ & $0,00 \mathrm{E}+00$ & $0,00 \mathrm{E}+00$ & $0,00 E+00$ & $0,00 E+00$ & $0,00 E+00$ & $0,00 E+00$ & $0,00 \mathrm{E}+00$ & $-3,75 E-27$ & $-6,13 \mathrm{E}-11$ \\
\hline $6,13 \mathrm{E}-11$ & $1,00 E+06$ & $0,00 \mathrm{E}+00$ & $0,00 \mathrm{E}+00$ & $0,00 E+00$ & $0,00 E+00$ & $0,00 E+00$ & $0,00 E+00$ & $0,00 E+00$ & $0,00 E+00$ & $-6,13 \mathrm{E}-11$ & $-1,00 \mathrm{E}+06$ \\
\hline$-1,00 E+06$ & $0,00 E+00$ & $2,35 E+06$ & $-3,54 E+05$ & $-1,00 E+06$ & $0,00 E+00$ & $0,00 E+00$ & $0,00 E+00$ & $-3,75 E-27$ & $-6,13 \mathrm{E}-11$ & $-3,54 E+05$ & $3,54 E+05$ \\
\hline $0,00 E+00$ & $0,00 E+00$ & $-3,54 \mathrm{E}+05$ & $1,35 \mathrm{E}+06$ & $0,00 E+00$ & $0,00 E+00$ & $0,00 E+00$ & $0,00 E+00$ & $-6,13 \mathrm{E}-11$ & $-1,00 \mathrm{E}+06$ & $3,54 \mathrm{E}+05$ & $-3,54 E+05$ \\
\hline $0,00 E+00$ & $0,00 E+00$ & $-1,00 E+06$ & $0,00 \mathrm{E}+00$ & $1,35 \mathrm{E}+06$ & $-3,54 E+05$ & $-3,75 E-27$ & $-6,13 \mathrm{E}-11$ & $-3,54 \mathrm{E}+05$ & $3,54 \mathrm{E}+05$ & $0,00 \mathrm{E}+00$ & $0,00 E+00$ \\
\hline $0,00 E+00$ & $0,00 E+00$ & $0,00 E+00$ & $0,00 \mathrm{E}+00$ & $-3,54 E+05$ & $1,35 \mathrm{E}+06$ & $-6,13 \mathrm{E}-11$ & $-1,00 \mathrm{E}+06$ & $3,54 \mathrm{E}+05$ & $-3,54 E+05$ & $0,00 \mathrm{E}+00$ & $0,00 E+00$ \\
\hline $0,00 E+00$ & $0,00 E+00$ & $0,00 E+00$ & $0,00 E+00$ & $-3,75 E-27$ & $-6,13 \mathrm{E}-11$ & $1,00 E+06$ & $6,13 \mathrm{E}-11$ & $-1,00 E+06$ & $0,00 \mathrm{E}+00$ & $0,00 E+00$ & $0,00 E+00$ \\
\hline $0,00 E+00$ & $0,00 E+00$ & $0,00 \mathrm{E}+00$ & $0,00 \mathrm{E}+00$ & $-6,13 \mathrm{E}-11$ & $-1,00 E+06$ & $6,13 \mathrm{E}-11$ & $1,00 \mathrm{E}+06$ & $0,00 E+00$ & $0,00 \mathrm{E}+00$ & $0,00 E+00$ & $0,00 E+00$ \\
\hline $0,00 E+00$ & $0,00 E+00$ & $-3,75 E-27$ & $-6,13 \mathrm{E}-11$ & $-3,54 E+05$ & $3,54 \mathrm{E}+05$ & $-1,00 E+06$ & $0,00 E+00$ & $2,35 E+06$ & $-3,54 E+05$ & $-1,00 E+06$ & $0,00 E+00$ \\
\hline $0,00 E+00$ & $0,00 E+00$ & $-6,13 \mathrm{E}-11$ & $-1,00 E+06$ & $3,54 E+05$ & $-3,54 E+05$ & $0,00 E+00$ & $0,00 E+00$ & $-3,54 E+05$ & $1,35 E+06$ & $0,00 E+00$ & $0,00 E+00$ \\
\hline$-3,75 E-27$ & $-6,13 \mathrm{E}-11$ & $-3,54 E+05$ & $3,54 \mathrm{E}+05$ & $0,00 E+00$ & $0,00 E+00$ & $0,00 E+00$ & $0,00 E+00$ & $-1,00 E+06$ & $0,00 E+00$ & $1,35 \mathrm{E}+06$ & $-3,54 E+05$ \\
\hline$-6,13 \mathrm{E}-11$ & $-1,00 \mathrm{E}+06$ & $3,54 \mathrm{E}+05$ & $-3,54 \mathrm{E}+05$ & $0,00 \mathrm{E}+00$ & $0,00 \mathrm{E}+00$ & $0,00 E+00$ & $0,00 E+00$ & $0,00 E+00$ & $0,00 \mathrm{E}+00$ & $-3,54 E+05$ & $1,35 \mathrm{E}+06$ \\
\hline
\end{tabular}


Conhecidos os valores dos deslocamentos, torna-se possível calcular as forças axiais das barras através da equação 22:

$$
\{q\}=[k]^{\prime}[T]\{D\}
$$

Em que $\{\mathrm{q}\}$ representa o vetor de carga nodal local de cada barra, [k]' a matriz de rigidez elementar, [T] a matriz de transformação de coordenadas globais para coordenadas locais e $\{D\}$ o vetor de deslocamentos globais. Assim podemos classificar a força em tração ou compressão.

Com o objetivo de realizar uma melhor análise da estrutura e tornar o processo mais dinâmico, os resultados obtidos também são ilustrados via programação em VBA. O programa, além de ilustrar a estrutura, também desenha a treliça de acordo com a intensidade das forças que ocorrem em cada barra, ou seja, a transparência no desenho de cada barra é dependente de sua intensidade. Outra função é a representação da estrutura deformada, em uma escala definida pelo próprio usuário.

A seguir, na figura 8 , apresenta-se o fluxograma para que os cálculos sejam realizados:

Figura 8 - Fluxograma

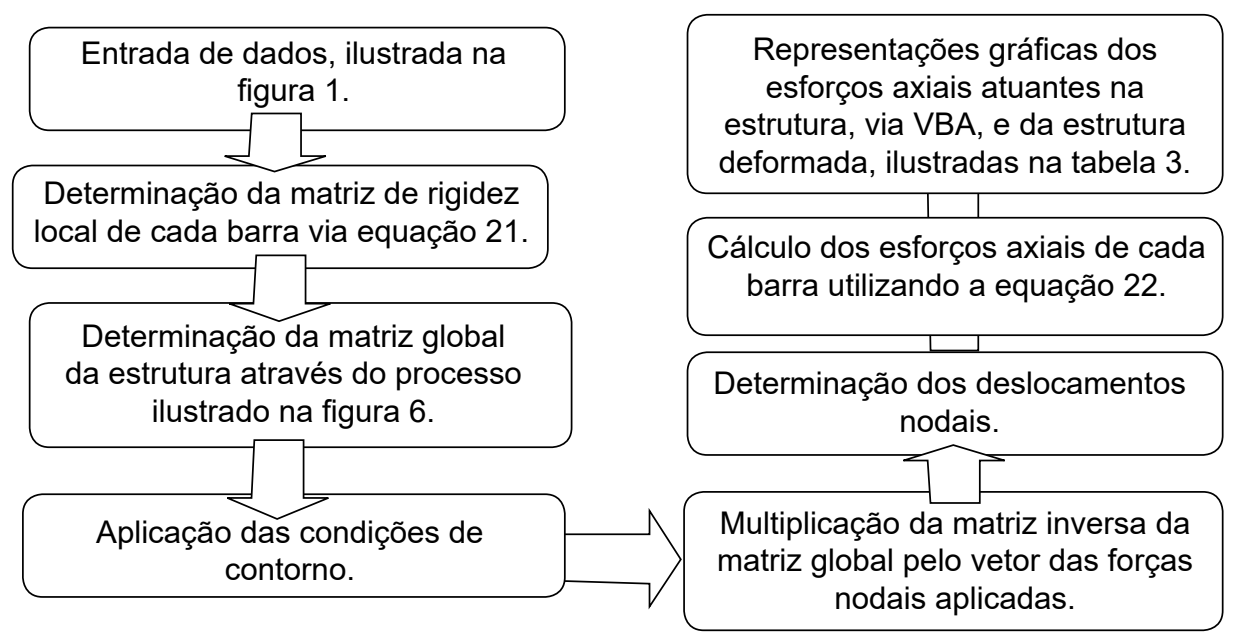

Para verificar a validade e a precisão do programa, foram comparados os resultados de diversos modelos de treliças, representadas na figura 9, obtidos pelo Microsoft Exce/® e pelo software de engenharia Ftool@ As treliças foram modeladas considerando que todas as barras são circulares, com área da seção de 10 $\mathrm{cm}^{2}$ e módulos de elasticidade de $1 \mathrm{MPa}$, e que os nós previamente indicados sofreram esforços de $100 \mathrm{kN}$, gerando os seguintes modelos: 
Figura 9 - Modelos de treliça estudados.

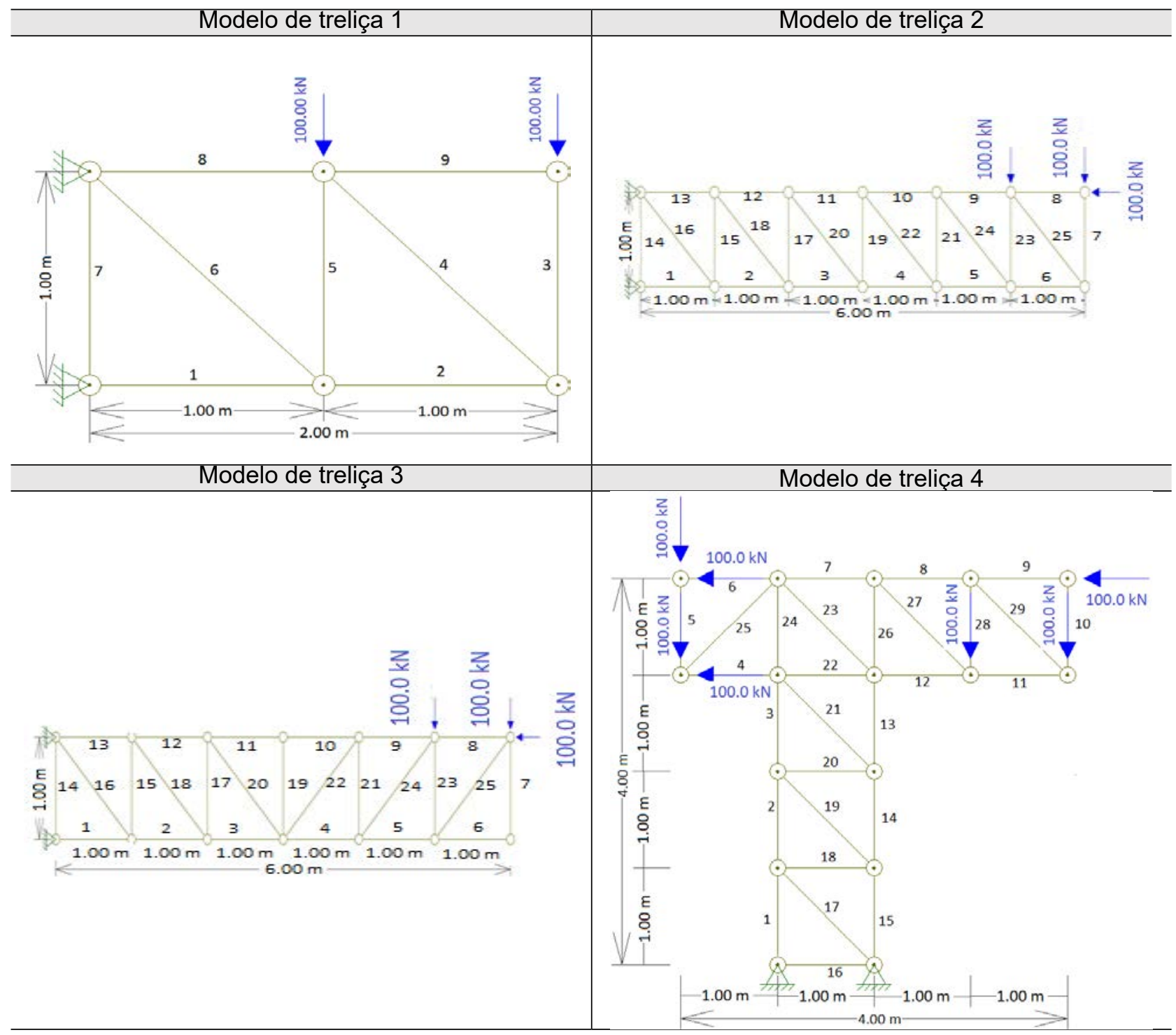

\section{Resultados}

Após a obtenção dos resultados foi gerada uma tabela para cada modelo de treliça, que comprovou a validade do programa, tendo se mantido a precisão dos valores dos esforços axiais em 4 casas decimais após a virgula, conforme a Tab (1):

Tabela 1 - Tabela de comparação de resultados para treliça 1.

\begin{tabular}{l|c|c|c|c|c|c|c|c|c}
\hline \multicolumn{1}{c|}{ Barra } & 1 & 2 & 3 & 4 & 5 & 6 & 7 & 8 & 9 \\
\hline Resultados & $-30,0000$ & $-10,0000$ & $-10,0000$ & 14,1421 & $-20,0000$ & 28,2843 & 0,0000 & 10,0000 & 0,0000 \\
\hline Ftool & $-30,0000$ & $-10,0000$ & $-10,0000$ & 14,1421 & $-20,0000$ & 28,2843 & 0,0000 & 10,0000 & 0,0000 \\
\hline
\end{tabular}

A planilha, além de ter obtido resultados validos, também gerou a representação da treliça, mostrando a intensidade das forças que ocorrem em cada barra e a estrutura deformada em escalas arbitradas. A representação da intensidade em cada barra ocorre por meio da aplicação de um gradiente de cores que expõe em vermelho a tração e em azul, compressão. A configuração da escala de cores auxilia a compreensão da intensidade, desde o esforço nulo (transparência total do elemento) até o esforço máximo (vermelho ou azul 
absoluto). O usuário ainda pode alterar a representação da configuração da estrutura deformada por meio de ajustes na escala em uma célula específica.

Na figura 10, nota-se que as barras 7 e 9 não estão sendo solicitadas, por isso as linhas são ocultadas na representação de intensidades.

Figura 10 - Resultados gráficos do modelo 1.

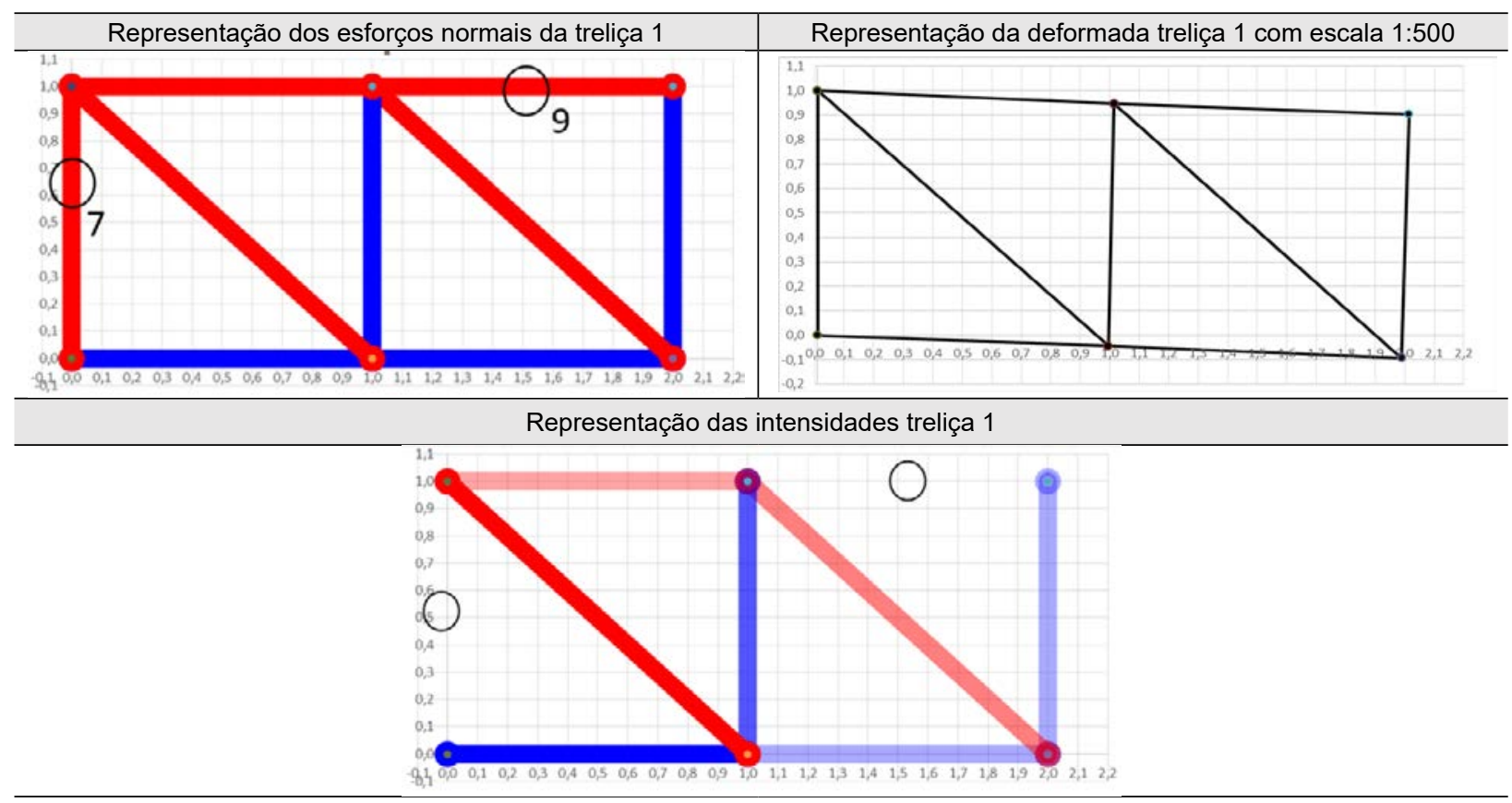

Na figura 11, as barras 1 e 13 sofrem os máximos esforços, sendo 110 kN de compressão e $80 \mathrm{kN}$ de tração.

Figura 11 - Resultados gráficos do modelo 2.

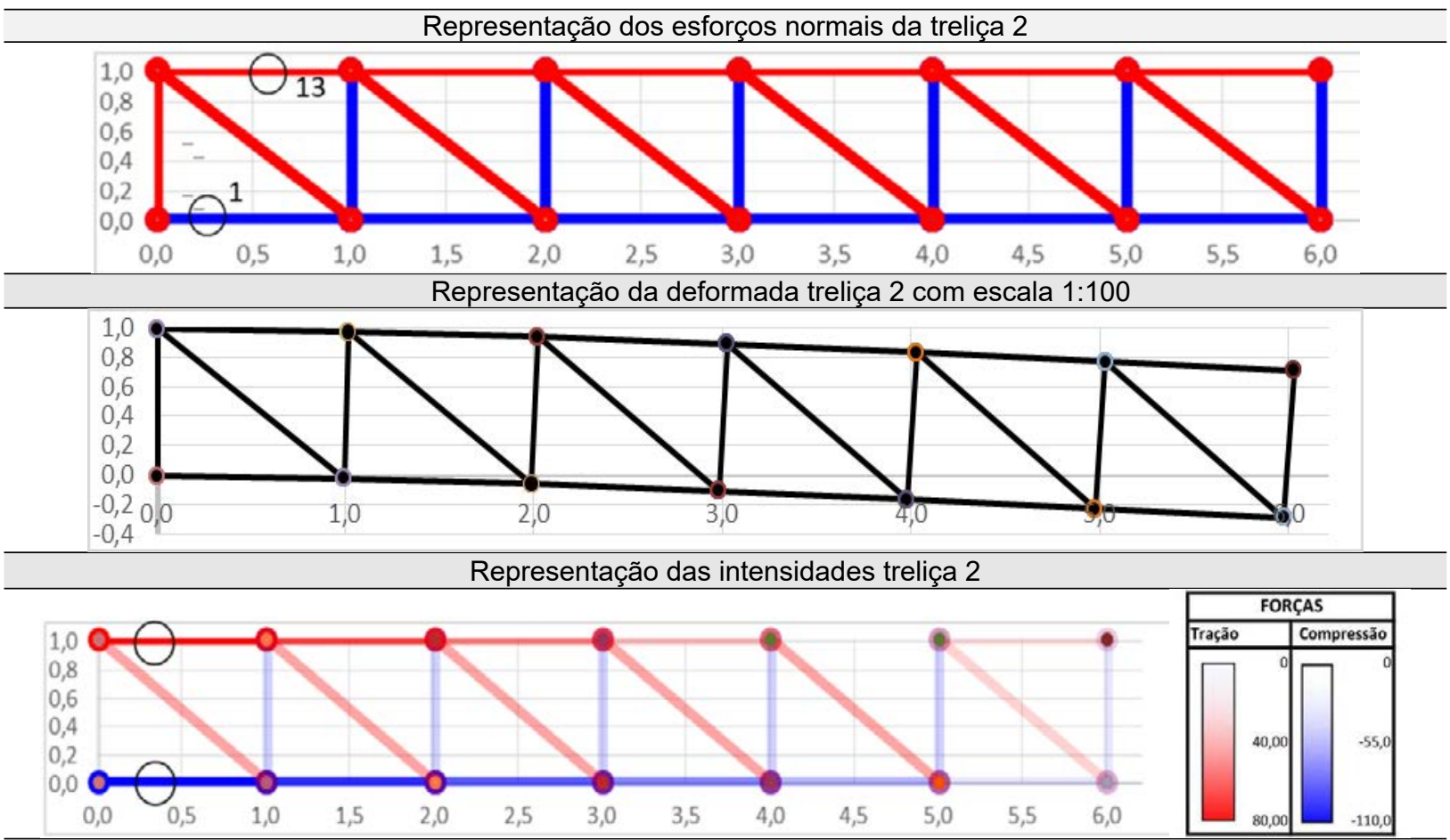


Na figura 12, observamos que os esforços máximos da treliça 3 são iguais aos da treliça 2, tendo somente alterações nas barras internas e a anulação dos esforços axiais na barra 19, a partir de onde ocorre a inversão das solicitações.

Figura 12 - Resultados gráficos do modelo 3.

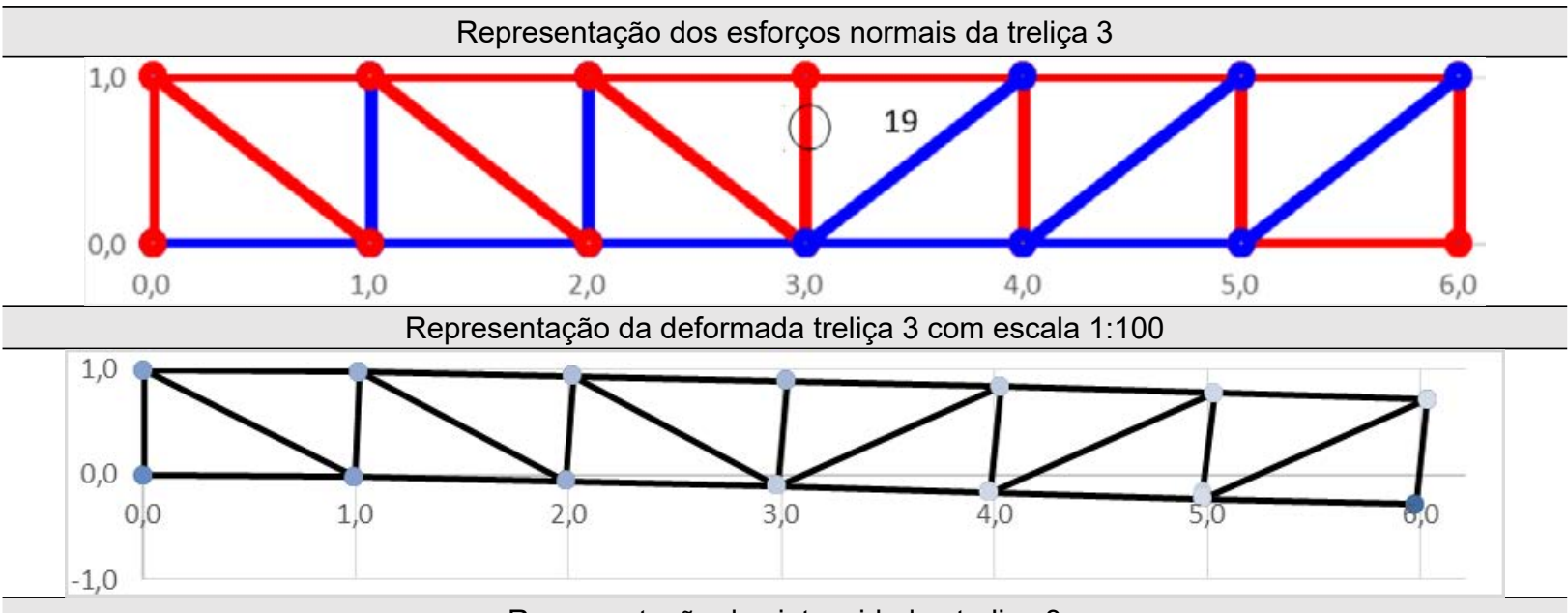

Representação das intensidades treliça 3

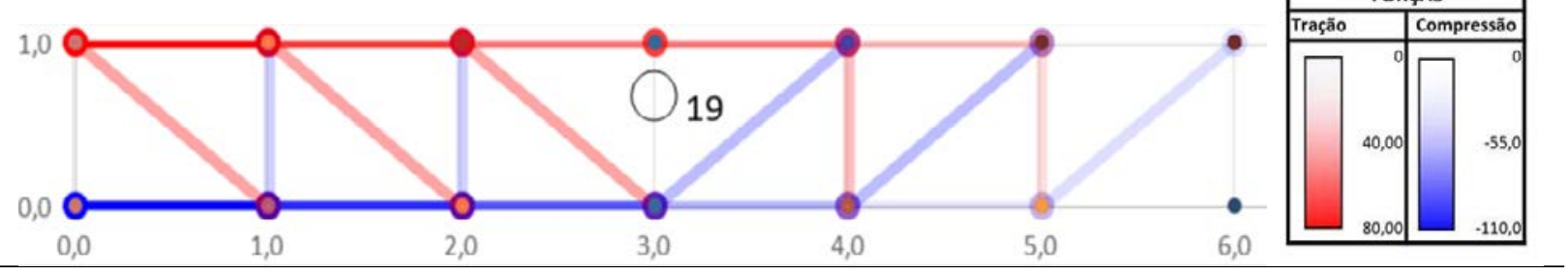

Na figura 13, o nó 10 teve um deslocamento igual aos valores obtidos pelo Ftool, de 1,11 mm no eixo $\mathrm{X}$ e de $6,03 \mathrm{~mm}$ no eixo $\mathrm{Y}$.

Figura 13 - Resultados gráficos do modelo 4.

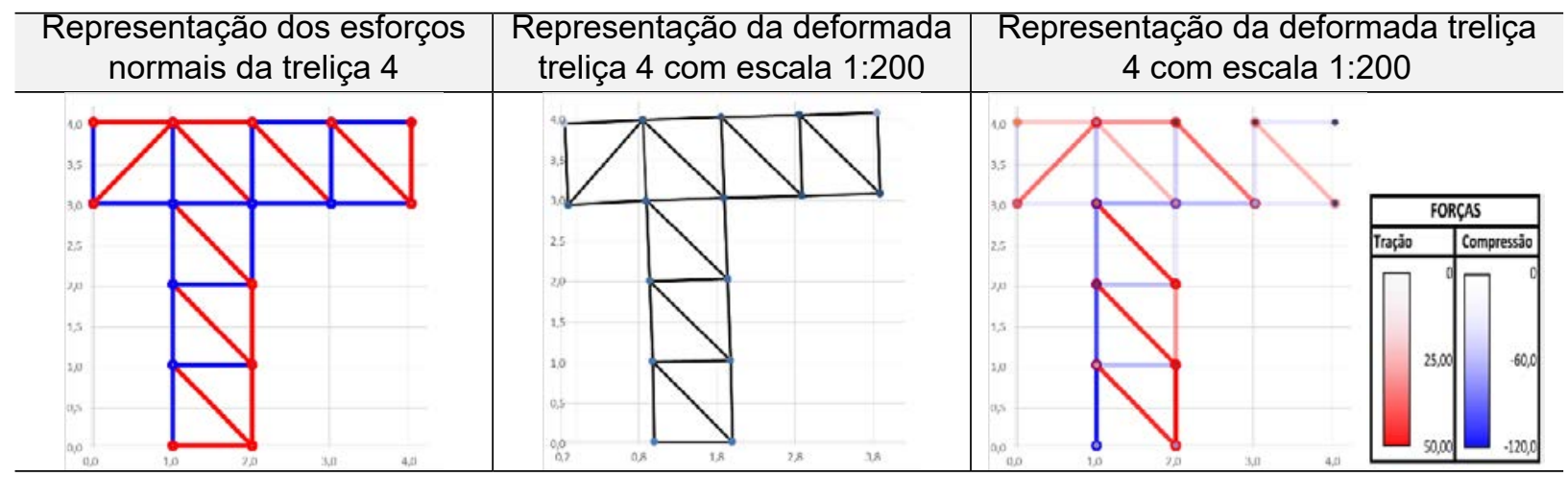

\section{Conclusão}

Observando-se a necessidade e a importância de conhecer as forças axiais nas barras de uma estrutura treliçada e de seus deslocamentos, visto que todo o dimensionamento e as etapas posteriores são dependentes desses valores calculados, a planilha apresentou resultados satisfatórios e precisos. Essa afirmação é baseada no êxito da comparação dos valores obtidos pelo Exce/ße dos valores obtidos pelo software educacional Ftool . $^{\circ}$ 
Os resultados gráficos tornam a análise da estrutura mais fácil para o usuário graças ao gradiente das cores, dependente da intensidade das tensões. Permite, também, analisar rapidamente as alterações das tensões e deslocamentos caso haja uma alteração nos dados de entrada.

O trabalho focou no estudo de treliças planas, sujeitas ou não a cargas nodais aplicadas, com enfoque no uso do Microsoft Exce/® para o ensino dos cálculos, apresentando uma forma mais didática e dinâmica comparada a outras maneiras de cálculo, representando os esforços e as deformações graficamente, por meio do VBA.

É indispensável o entendimento da teoria para que os resultados possam ser analisados da melhor forma, não se prendendo somente ao bom uso dos programas de cálculo. Nesse contexto, a planilha exibe todo o memorial de cálculo para ser estudado o passo a passo da construção dos resultados, o que configura uma opção de ferramenta educacional.

\section{Referências}

BATHE, K. J. Finite element procedures in engineering analysis. New Jersey: Prentice-Hall, 1982.

FTOOL. Two-dimensional frame analysis tool. Disponível em: https://www.ftool.com.br/Ftool/. Acesso em: 02 nov. 2018.

GODOI, R. M.; VANALLI, L.; SILVA, S. S. Análise de estruturas utilizando o software excel através do método da rigidez direta. In: 26 ENCONTRO ANUAL DE INICIAÇÃO CIENTÍFICA - UEM., Maringá, 2017. Disponível em: <http://www.eaic.uem.br/eaic2017/anais/artigos/2077.> Acessado em: Nov. 2018.

MARTHA, Luiz F. Análise de estruturas: conceitos e métodos básicos. 1. ed. Rio de Janeiro: Elsevier, 2010.

NOGUEIRA, L. G. O.; BEZERRA, E. M. F. Ferramenta em ambiente excel para análise estrutural de treliças espaciais pelo método dos elementos finitos. In: IBERIAN LATIN-AMERICAN CONGRESS ON COMPUTATIONAL METHODS IN ENGINEERING, 38., Florianopolis, nov., 2017. Proceedings [...]. Disponível em: https://ss/4799.websiteseguro.com/swge5/PROCEEDINGS/PDF/CILAMCE2017-1280.pdf. Acesso em: 02 nov. 2018.

SILVA NETO, G. C. da; LOPES, R. C.; LOPES, A. P. O método dos elementos finitos em treliças planas na disciplina de mecânica computacional. In: CONGRESSO BRASILEIRO DE EDUCAÇÃO EM ENGENHARIA, 25., Curitiba, 2007. Anais [...]. Disponível em: http://www.abenge.org.br/cobenge/ arquivos/12/artigos/434-Gustavo\%20Cunha.pdf. Acesso em: 02 nov. 2018.

SORIANO, H. L.; LIMA, S. de S. Método de elementos finitos em análise de estruturas. 48. ed. São Paulo: EdUSP, 2003. 


\section{Sobre os autores}

\section{Charles Jaster de Oliveira}

Mestre em Engenharia de Estruturas pela Universidade de São Paulo (EESC, USP, 2012). Graduado em Engenharia Civil pela Universidade Federal do Paraná (UFPR, 2009). Professor assistente do curso de Engenharia Civil e Arquitetura da Universidade Positivo. Tem experiência profissional na área de Engenharia Civil, com ênfase em estruturas. Tem atuado principalmente nos seguintes temas: método dos elementos finitos, otimização topológica e ensino de estruturas na Engenharia Civil e Arquitetura.

\section{Luna Ollin Steffen}

Mestre em Engenharia Civil pela Universidade Tecnológica Federal do Paraná (UTFPR, 2018). Graduada em Engenharia Civil pela Universidade Tecnológica Federal do Paraná (UTFPR, 2015). Técnica em Construção Civil pela Universidade Tecnológica Federal do Paraná (UTFPR, 2010). Possui experiência na construção civil, tendo trabalhado com projetos estruturais, fiscalização de obras, gerenciamento de vendas imobiliárias e execução de obras e reformas. Atualmente, é professora nos cursos de Engenharia Civil e Engenharia Ambiental.

\section{Guilherme Macedo Vogel}

Graduando do curso de Engenharia Civil da Universidade Positivo.

\section{Raphael Brandalise Nunes}

Graduando do curso de Engenharia Civil da Universidade Positivo.

\section{Gabriel Martins dos Santos}

Graduando do curso de Engenharia Civil da Universidade Positivo.

Recebido em: 01.09.2019

Aceito em: 24.09.2019 